\title{
Types and Quantifiers in SHRUTI - a connectionist model of rapid reasoning and relational processing
}

\author{
Lokendra Shastri \\ International Computer Science Institute, Berkeley CA 94704, USA, \\ shastri@icsi.berkeley.edu, \\ WWW home page: http://icsi.berkeley/ shastri
}

\begin{abstract}
In order to understand language, a hearer must draw inferences to establish referential and causal coherence. Hence our ability to understand language suggests that we are capable of performing a wide range of inferences rapidly and spontaneously. This poses a challenge for cognitive science: How can a system of slow neuron-like elements encode a large body of knowledge and perform inferences with such speed? SHRUTI attempts to answer this question by demonstrating how a neurally plausible network can encode a large body of semantic and episodic facts, and systematic rule-like knowledge, and yet perform a range of inferences within a few hundred milliseconds. This paper describes a novel representation of types and instances in SHRUTI that supports the encoding of rules and facts involving types and quantifiers, enables sHRUTI to distinguish between hypothesized and asserted entities, and facilitates the dynamic instantiation and unification of entities during inference.
\end{abstract}

\section{Introduction}

In order to understand language, a hearer must draw inferences to establish referential and causal coherence, generate expectations, make predictions, and recognize the speaker's intent. Hence our ability to understand language suggests that we are capable of performing a wide range of inferences rapidly, spontaneously and without conscious effort - as though they are a reflex response of our cognitive apparatus. In view of this, such reasoning has been described as reflexive reasoning [22]. This remarkable human ability poses a challenge for cognitive science and computational neuroscience: How can a system of slow neuron-like elements encode a large body of systematic knowledge and perform a wide range of inferences with such speed?

The neurally plausible (connectionist) model sHRUTi attempts to address the above challenge. It demonstrates how a network of neuron-like elements could encode a large body of structured knowledge and perform a variety of inferences within a few hundred milliseconds [3][22][14][23][20].

SHRUTI suggests that the encoding of relational information (frames, predicates, etc.) is mediated by neural circuits composed of focal-clusters and the 
dynamic representation and communication of relational instances involves the transient propagation of rhythmic activity across these clusters. A role-entity binding is represented within this rhythmic activity by the synchronous firing of appropriate cells. Systematic mappings - and other rule-like knowledge - are encoded by high-efficacy links that enable the propagation of rhythmic activity across focal-clusters, and a fact in long-term memory is a temporal pattern matcher circuit.

The possible role of synchronous activity in dynamic neural representations has been suggested by other researchers (e.g., [28]), but shruTi offers a detailed computational account of how synchronous activity can be harnessed to solve problems in the representation and processing of high-level conceptual knowledge. A rich body of neurophysiological evidence has emerged suggesting that synchronous activity might indeed play an important role in neural computation [26] and several models using synchrony to solve the binding problem during inference have been developed (e.g., [9]). ${ }^{1}$

As an illustration of SHRUTI's inferential ability consider the following narrative: "John fell in the hallway. Tom had cleaned it. He got hurt." Upon being presented with the above narrative ${ }^{2}$ SHRUTI reflexively infers the following: ${ }^{3}$ Tom had mopped the floor. The floor was wet. John was walking in the hallway. John slipped and fell because the floor was wet. John got hurt because he fell.

Notice that SHRUTi draws inferences required to establish referential and causal coherence. It explains John's fall by making the plausible inference that John was walking in the hallway and he slipped because the floor was wet. It also infers that John got hurt because of the fall. Moreover, it determines that "it" in the second sentence refers to the hallway, and that "He" in the third sentence refers to John, and not to Tom.

The representational and inferential machinery developed in sHRUTi can be applied to other problems involving relational structures, systematic but contextsensitive mappings between such structures, and rapid interactions between persistent and dynamic structures. The sHruti model meshes with the "Neural Theory of Language" project [4] on language acquisition and provides neurally plausible solutions to several representational and computational requirements arising in the project. The model also offers a plausible framework for realizing the "Interpretation as Abduction" approach to language understanding described in [8]. Moreover, sHRUTI's representational machinery has been extended to realize control and coordination mechanisms required for modeling actions and reactive plans [24].

\footnotetext{
${ }^{1}$ For other solutions to the binding problem within a structured connectionist framework see [11][5][27].

2 Each sentence in the narrative is conveyed to SHRUTI as a set of dynamic bindings (see Section 4). The sentences are presented in the order of their occurrence in the narrative. After each sentence is presented, the network is allowed to propagate activity for a fixed number of cycles.

${ }^{3}$ A detailed discussion of this example appears in [25].
} 
This paper describes a novel representation of types and instances in SHRUTI. This representation supports the encoding of rules and facts involving types and quantifiers, and at the same time allows SHRUTI to distinguish between hypothesized entities and asserted entities. This in turn facilitates the dynamic instantiation and unification of entities and relational instances during inference. For a detailed description of various aspects of SHRUTI's representational machinery refer to [22][20][25].

The rest of the chapter is organized as follows: Section 2 provides an overview of how relational knowledge is encoded in sHRuti. Section 3 discusses the representation of types and instances. Section 4 describes the representation of dynamic bindings. Section 5 explains how phase separation between incompatible entities is enforced in the type hierarchy via inhibitory mechanisms, and how phases are merged to unify entities. Section 6 describes the associative potentiation of links in the type hierarchy. Next, Section 7 reviews the encoding of facts, and Section 8 outlines the encoding of rules (or mappings) between relational structures. A simple illustrative example is presented in Section 9.

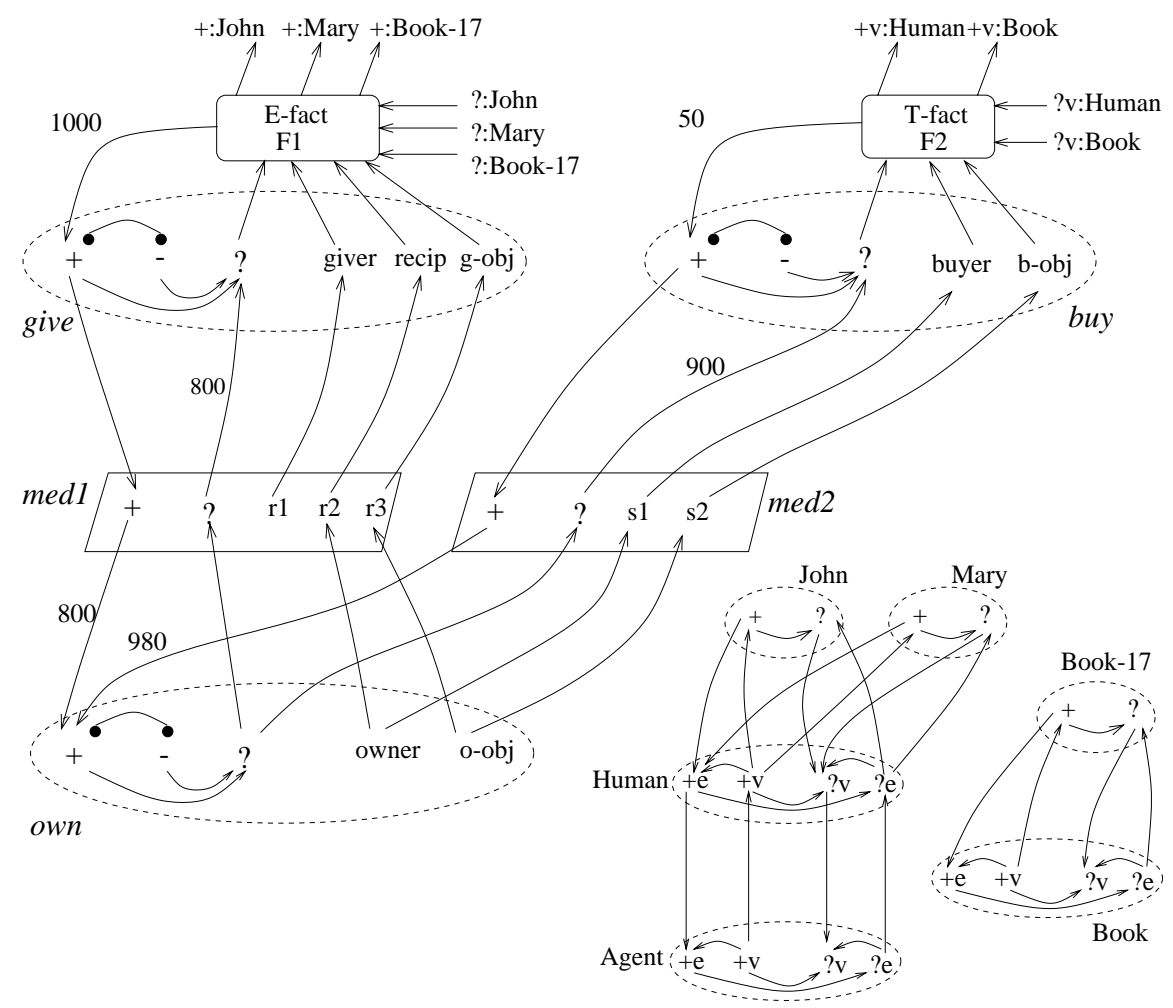

Fig. 1. An overview of sHRUTI's representational machinery. 


\section{An Overview of SHRUTI's Representational Machinery}

All long-term (persistent) knowledge is encoded in SHRUTI via structured networks of nodes and links. Such long-term knowledge includes generic relations, instances, types, general rules, and specific facts. In contrast, dynamic aspects of knowledge are represented via the activity of nodes, the propagation of activity along excitatory and inhibitory links, and the integration of incident activity at nodes. Such dynamic knowledge includes active (dynamic) facts and bindings, propagation of bindings, fusion of evidence, competition among incompatible entities, and the development of coherence.

Figure 1 provides an overview of some of the key elements of sHRUTI's representational machinery. The network fragment shown in the figure depicts a partial encoding of the following rules, facts, instances, and types:

1. $\forall(x:$ Agent $y:$ Agent $z:$ Thing) give $(x, y, z) \Rightarrow$ own $(y, z)[800,800]$;

2. $\forall(x:$ Agent $y$ :Thing) buy $(x, y) \Rightarrow$ own $(x, y)[900,980]$;

3. EF: give (John, Mary, Book-17) [1000];

4. TF: $\forall(x: H u m a n ~ y: B o o k)$ buy $(x, y)[50]$;

5. is-a(John, Human);

6. is-a(Mary, Human); 7. is-a(Human, Agent); 8. is-a(Book-17, Book).

Item (1) is a rule which captures a systematic relationship between giving and owning. It states that when an entity $x$ of type Agent, gives an entity $z$ of type Thing, to an entity $y$ of type Agent, then the latter comes to own it. Similarly, item (2) is a rule which states that whenever any entity of the type Agent buys something, it comes to own it. The pair of weights [a,b] associated with a rule have the following interpretation: $a$ indicates the degree of evidential support for the antecedent being the probable cause (or explanation) of the consequent, and $b$ indicates the degree of evidential support for the consequent being a probable effect of the antecedent. "Item (3) corresponds to a long-term "episodic" fact (or E-fact) which states that John gave Mary a specific book (Book-17). Item (4) is a long-term "taxon" fact (or T-fact) which states that the prior evidential support for a given (random) human buying a given (random) book is 50 . Item (5) states that John is a human. Similarly, items (6-8).

Given the above knowledge, sHRUTi can rapidly draw inferences of the following sort within a few hundred milliseconds ${ }^{5}$ (numbers in [] indicate strength of inference):

1. own (Mary, Book-17) [784];

Mary owns a particular book (referred to as Book-17).

\footnotetext{
${ }^{4}$ Weights in SHRUTI lie in the interval [0,1000]. The mapping of probabilities and evidential supports to weights in SHRUTI is non-linear and loosely defined. The initial weights can be set approximately, and subsequently fine tuned to model a given domain via learning.

${ }^{5}$ The time required for drawing an inference is estimated by $c * \pi$, where $c$ is the number of cycles of rhythmic activity it takes SHRUTI to draw an inference (see Section 9), and $\pi$ is the period of rhythmicity. A plausible value of $\pi$ is 25 milliseconds [22].
} 
2. $\exists x: B o o k$ own (Mary, $x)$ [784];

Mary owns a book.

3. $\exists(x:$ Agent $y$ :Thing) own $(x, y)$ [784];

Some agent owns something.

4. buy(Mary,Book-1) [41];

Mary bought a particular book (referred to as Book-1).

5. is-a(Mary, Agent);

Mary is an agent.

Figure 2 depicts a schematized response of the SHRUTI network shown in Figure 1 to the query "Does Mary own a book?" ( $\exists x$ :Book own(Mary, $x)$ ?). We will revisit this activation trace in Section 9 after we have reviewed SHRUTI's representational machinery, and discussed the encoding of instances and types in more detail. For now it suffices to observe that the query is conveyed to the network by activating appropriate "?" nodes (?:own, ?:Mary and ?e:Book) and appropriate role nodes (owner and $o-o b j$ ). This leads to a propagation of activity in the network which eventually causes the activation of the nodes $+:$ own and $+:$ Book-17. This signals an affirmative answer (Yes, Mary owns Book-17). Note that bindings between roles and entities are expressed by the synchronous activation of bound role and entity nodes.

\subsection{Different node types and their computational behavior}

Nodes in SHRUTI are computational abstractions and correspond to small ensembles of cells. Moreover, a connection from a node A to a node B corresponds to several connections from cells in the A ensemble to cells in the B ensemble.

SHRUTI makes use of four node types: m- $\rho$-nodes, $\tau$-and nodes, $\tau$-or nodes of type 1 ), and $\tau$-or nodes of type 2 . This classification is based on the computational properties of nodes, and not on their functional or representational role. In particular, nodes serving different representational functions can be of the same computational type. The computational behavior of $\mathrm{m}-\rho$-nodes and $\tau$-and nodes is described below:

m- $\rho$ nodes: An m- $\rho$ node with threshold $n$ becomes active and fires upon receiving $n$ synchronous inputs. Here synchrony is defined relative to a window of temporal integration $\omega$. Thus all inputs arriving at a node with a lead/lag of no more than $\omega$, are deemed to be synchronous. Thus an $\mathrm{m}-\rho$ node $A$ receiving above-threshold periodic inputs from m- $\rho$ nodes $B$ and $C$ (where $B$ and $C$ may be firing in different phases) will respond by firing in phase with both $B$ and $C$. A similar node type has been described in [15].

A scalar level (strength) of activity is associated with the response of an $\mathrm{m}-\rho$ node. ${ }^{6}$ This level of activity is computed by the activation combination function (ECF) associated with the node. Some ECFs used in the past are sum, max, and sigmoid. Other combination functions are under investigation [25].

${ }^{6}$ The response-level of a $\mathrm{m}-\rho$ node in a phase can be governed by the number of cells in the node's cluster firing in that phase. 


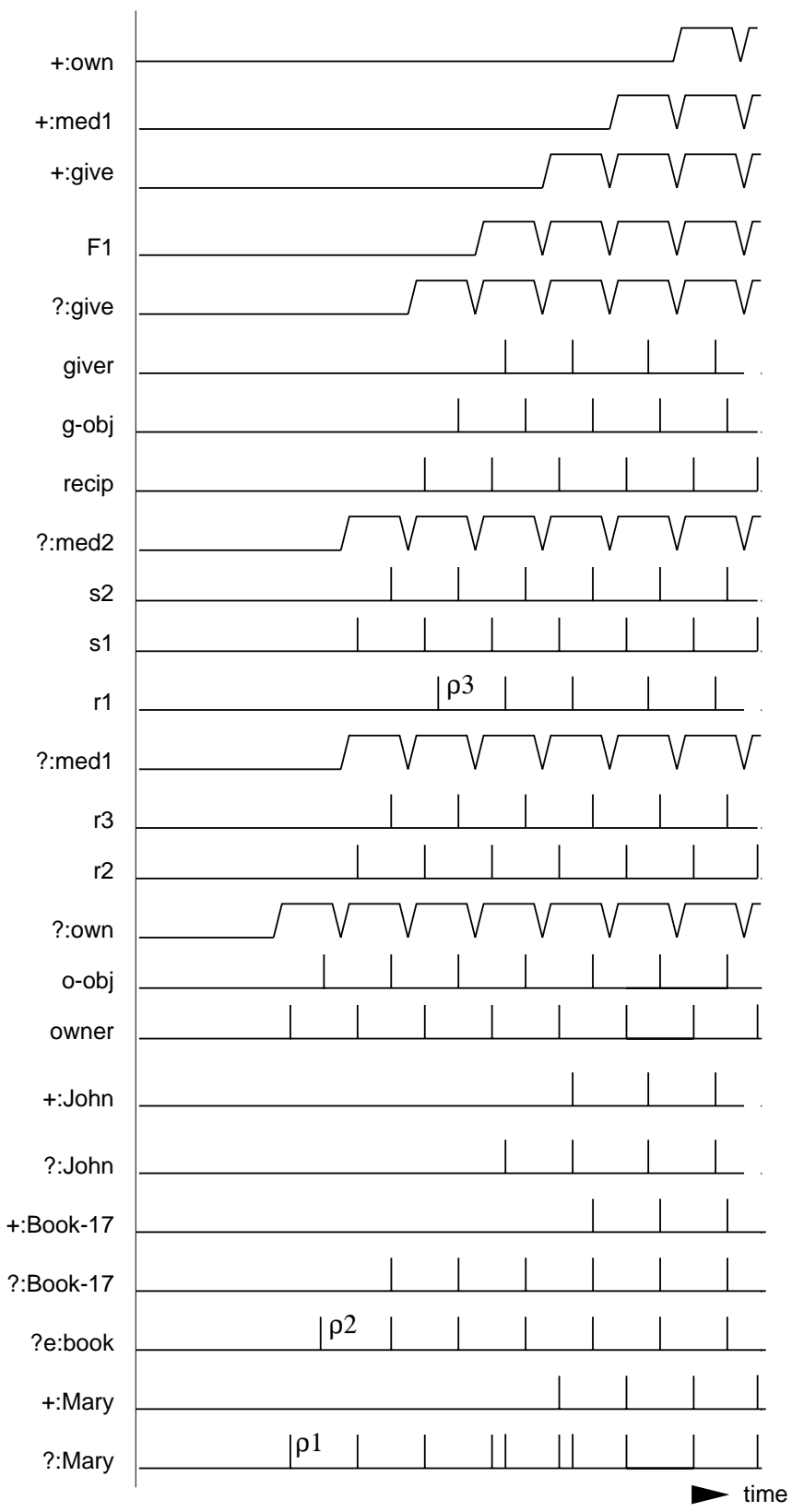

Fig. 2. A schematized activation trace of selected nodes for the query own(Mary,Book17)? 
$\tau$-and nodes: A $\tau$-and node becomes active on receiving an uninterrupted and above-threshold input over an interval $\geq \pi_{\max }$, where $\pi_{\max }$ is a system parameter. Computationally, this sort of input can be idealized as a pulse whose amplitude exceeds the threshold, and whose duration is greater than or equal to $\pi_{\text {max }}$. Physiologically, such an input may be identified with a high-frequency burst of spikes. Thus a $\tau$-and node behaves like a temporal and node and becomes active upon receiving adequate and uninterrupted inputs over an interval $\pi_{\text {max }}$. Upon becoming active, such a node produces an output pulse of width $\geq$ $\pi_{\max }$. The level of output activation is determined by the ECF associated with the node for combining the weighted inputs arriving at the node.

The model also makes use of inhibitory modifiers that can block the flow of activation along a link. This blocking is phasic and lasts only for a duration $\omega$.

\subsection{Encoding of relational structures}

Each relation (in general, a frame or a predicate) is represented by a focal-cluster which as an anchor for the complete encoding of a relation. Such focal-clusters are depicted as dotted ellipses in Figure 1. The focal-cluster for the relation give is depicted toward the top and the left of Figure 1. For the purpose of this example, it is assumed that give has only three roles: giver, recipient and giveobject. Each of these roles is encoded by a separate node labeled giver, recip and $g$-obj, respectively. The focal-cluster of give also includes an enabler node labeled ? and two collector nodes labeled + and - . The positive and negative collectors are mutually inhibitory (inhibitory links are depicted by filled blobs). In general, the focal-cluster for an $n$-place relation contains $n$ role nodes, one enabler node, one positive collector node and one negative collector node. We will refer to the enabler, the positive collector, and the negative collector of a relation $P$ as $?: P$, $+: P$, and $-: P$, respectively. The collector and enabler nodes of relations behave like $\tau$-and nodes. Role nodes and the collector and enabler nodes of instances behave like $m-\rho$ nodes.

Semantic import of enabler and collector nodes. Assume that the roles of a relation $P$ have been dynamically bound to some fillers and thereby represent an active instance of $P$ (we will see how this is done, shortly). The activation of the enabler ?:P means that the system is seeking an explanation for the active instance of $P$. In contrast, the activation of the collector $+: P$ means that the system is affirming the active instance of $P$. Similarly, the activation of the collector -:P means that the system is affirming the negation of the active instance of $P$. The activation levels of $?: P,+: P$ and $-: P$ signifies the strength with which information about $P$ is being sought, believed, or disbelieved, respectively.

For example, if the roles giver, recipient and object are dynamically bound to John, Mary, and a book, respectively, then the activation of ?:give means that the system is asking whether "John gave Mary a book" matches a fact in memory, or whether it can be inferred from what is known. In contrast, the activation of $+: P$ with the same role bindings means that the system is asserting "John gave Mary a book". 
Degrees of belief: support, no information and contradiction. The levels of activation of the positive and negative collectors of a relation measure the effective degree of support offered by the system to the currently active relational instance. Thus the activation levels of the collectors $+: P$ and $-: P$ encode a graded belief ranging continuously from no on the one extreme (only -:P is active), to yes on the other (only $+: P$ is active), and don't know in between (neither collector is very active). If both the collectors receive comparable and strong activation then a contradiction is indicated.

Significance of collector to enabler connections. Links from the collector nodes to the enabler node of a relation convert a dynamic assertion of a relational instance into a query about the assertion. Thus the system continually seeks an explanation for active assertions. The weight on the link from $+: P($ or $-: P)$ to $?: P$ is a sum of two terms. The first term is proportional to the system's propensity for seeking explanations - the more skeptical the system, the higher the weight. The second term is inversely proportional to the probability of occurrence of a positive (or negative) instance of $P$ - the more unlikely a fact, the more intense the search for an explanation.

The links from the collectors of a relation to its enabler also create positive feedback loops of activation and thereby create stable coalitions of active cells under appropriate circumstances. If the system seeks an explanation for an instance of $P$ and finds support for this instance, then a stable coalition of activity arises consisting of ?:P, other ensembles participating in the explanation, $+: P$ and finally ?:P. Such activity leads to priming (see Section 6), and the formation of episodic memories (see [19,21]).

\section{$3 \quad$ Encoding instances and types}

The encoding of types and instances is illustrated in Figure 3 . The focal-cluster of each entity consists of a ? and a + node. In contrast, the focal-cluster of each type consists of a pair of ? nodes (?e and $? v)$ and a pair of + nodes $(+e$ and $+v$ ). While the nodes $+v$ and $? v$ participate in the expression of knowledge (facts and attributes) involving the whole type, the nodes $+e$ and $? e$ participate in the encoding of knowledge involving particular instances of the type. Thus nodes $v$ and $e$ signify universal and existential quantification, respectively. All nodes participating in the representation of types are $m-\rho$ nodes.

\subsection{Interconnections within focal-clusters of instances and types}

The interconnections shown in Figure 3 among nodes within the focal-cluster of an instance and among nodes within the focal-cluster of a type lead to the following functionality ( $I$ refers to an instance, $T 1$ refers to a type):

- Because of the link from $+: I$ to ?:I, any assertion about an instance leads to a query or a search for a possible explanation of the assertion. 
- Because of the link from +v:T1 to +e:T1, any assertion about the type leads to the same assertion being made about an unspecified member of the type (e.g., "Humans are mortal" leads to "there exists a mortal human"). ${ }^{7}$

- Because of the link from +v:T1 to ?v:T1, any assertion about the whole type leads to a query or search for a possible explanation of the assertion (e.g., the assertion "Humans are mortal" leads to the query "Are humans mortal?").

- Because of the link from +e:T1 to ?e:T1, any assertion about an instance of the type leads to a query or search for a possible instance that would verify the assertion (e.g., the assertion "There is a human who is mortal" to the query "Is there is a human who is mortal?").

- Because of the link from ?e:T1 to ?v:T1, any query or search for an explanation about a member of the type leads to a query about the whole type (one way of determining whether "A human is mortal" is to find out whether "Humans are mortal").

- Moreover, paths formed by the above links lead to other behaviors. For example, given the path from +v:T1 to ?e:T1, any assertion about the whole type leads to a query or search for an explanation of the assertion applied to a given subtype/member of the type (e.g., "Humans are mortal" leads to the query "Is there a human who is mortal?").

Note that the closure between the "?" and "+" nodes is provided by the matching of facts (see Section 7).

\subsection{The interconnections between focal-clusters of instances and types}

The interconnections between nodes in the focal-clusters of instances and types lead to the following functionality:

- Because of the link from $+\mathrm{v}: T 1$ to $+: I$, any assertion about the type $T 1$ leads to the same assertion about the instance $I$ ("Humans are mortal" leads to "John is mortal").

- Because of the link from $+: I$ to $+\mathrm{e}: T 1$, any assertion about $I$ leads to the same assertion about a member of $T 1$ ("John is mortal" leads to "A human is mortal").

- Because of the link from ?:I to ?v:T1, a query about $I$ leads to a query about $T 1$ as a whole (one way of determining whether "John is mortal" is to determine whether "Humans are mortal").

- Because of the link from ?e:T1 to ?:I, a query about a member of $T 1$ leads to a query about I (one way of determining whether "A human is mortal" is to determine whether "John is mortal").

Similarly, interconnections between sub- and supertypes lead to the following functionality.

${ }^{7}$ SHRUTI infers the existence of a mortal human given that all humans are mortal, though this is not entailed in classical logic. 
- Because of the link from $+\mathrm{v}: T 2$ to $+\mathrm{v}: T 1$, any assertion about the supertype $T 2$ leads to the same assertion about the subtype $T 1$ ("Agents can cause change" leads to "Humans can cause change").

- Because of the link from +e:T1 to +e:T2, any assertion about a member of $T 1$ leads to the same assertion about a member of $T 2$ ("Humans are mortal" leads to "mortal agents exist").

- Because of the link from ?v:T1 to ?v:T2, a query about $T 1$ as a whole leads to a query about $T 2$ as a whole (one way of determining whether "Humans are mortal" is to determine whether "Agents are mortal").

- Because of the link from ?e:T2 to ?e:T1, a query about a member of $T 2$ leads to a query about a member of $T 1$ (one way of determining whether "an Agent is mortal" is to determine whether "a Human is mortal").

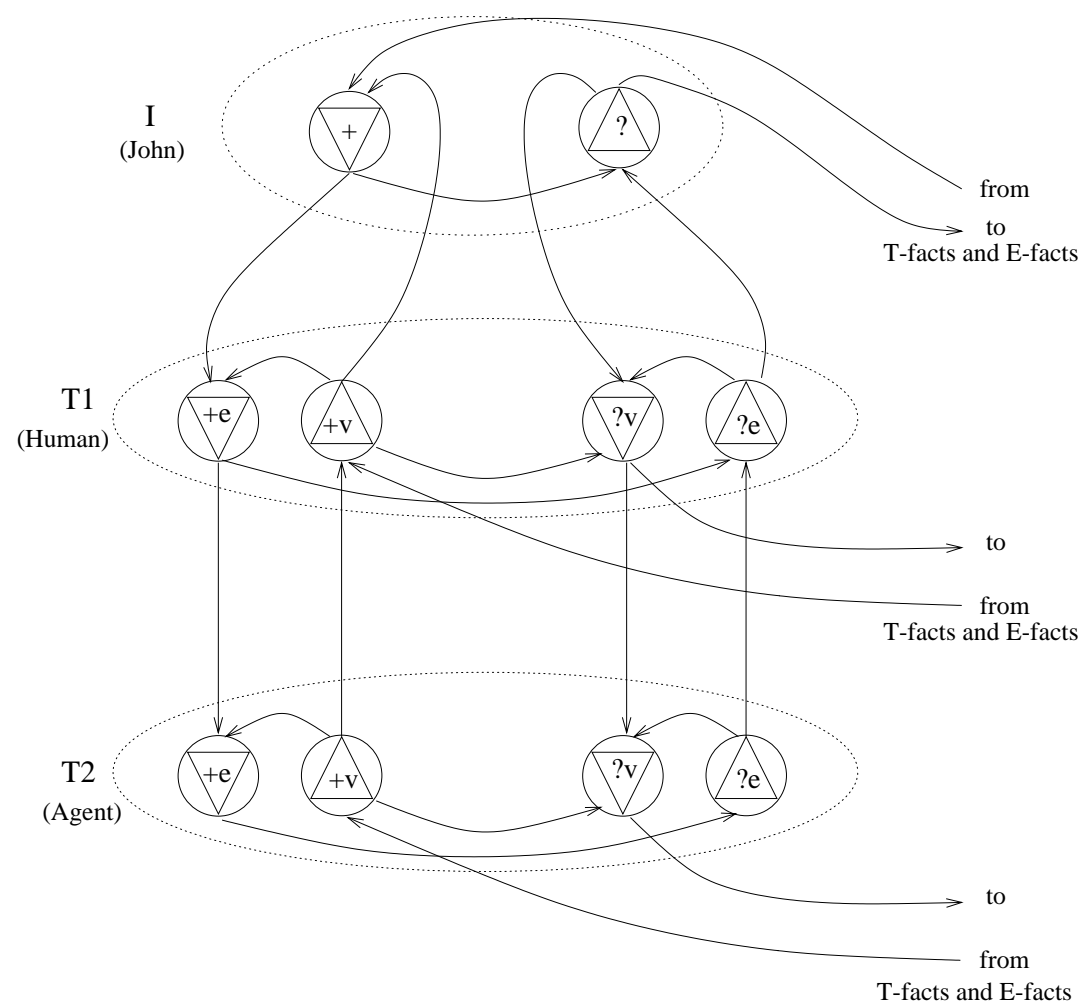

Fig. 3. The encoding of types and (specific) instances. See text for details. 


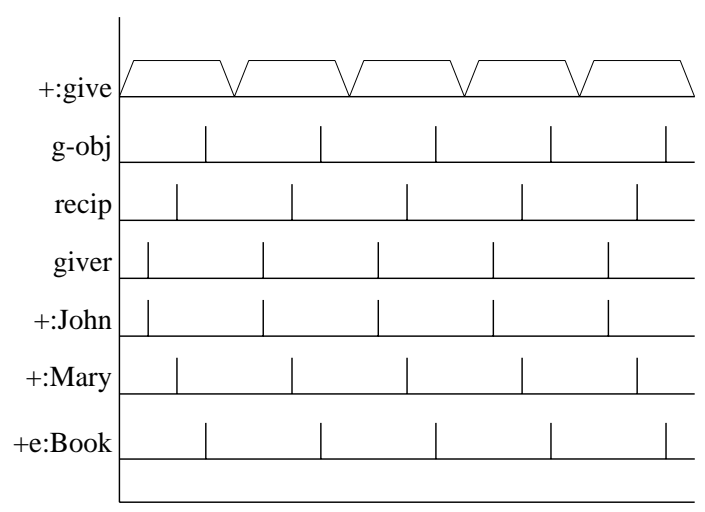

Fig. 4. The rhythmic activity representing the dynamic bindings give(John, Mary, aBook). Bindings are expressed by the synchronous activity of bound role and entity nodes.

\section{Encoding of dynamic bindings}

The dynamic encoding of a relational instance corresponds to a rhythmic pattern of activity wherein bindings between roles and entities are represented by the synchronous firing of appropriate role and entity nodes. With reference to Figure 1, the rhythmic pattern of activity shown in Figure 4 is the dynamic representation of the relational instance (give: $\langle$ giver $=$ John $\rangle$, 〈recipient=Mary), $\langle$ give-object=a-Book $\rangle$ ) (i.e., "John gave Mary a book"). Observe that the collector ensembles $+:$ John, + :Mary and $+e:$ Book are firing in distinct phases, but in phase with the roles giver, recip, and $g$-obj, respectively. Since $+:$ give is also firing, the system is making an assertion. The dynamic representation of the query "Did John give Mary a book?" would be similar except that the enabler node would be active and not the collector node.

The rhythmic activity underlying the dynamic representation of relational instances is expected to be highly variable, but it is assumed that over short durations - ranging from a few hundred milliseconds to about a second such activity may be viewed as being composed of $k$ interleaved quasi-periodic activities where $k$ equals the number of distinct entities filling roles in active relational instances. The period of this transient activity is at least $k * \omega_{\text {int }}$ where $\omega_{\text {int }}$ is the window of synchrony, i.e., the amount by which two spikes can lead/lag and still be treated as being synchronous. As speculated in [22], the activity of role and entity cells engaged in dynamic bindings might correspond to $\gamma$ band activity $(\sim 40 \mathrm{~Hz})$.

\section{$5 \quad$ Mutual exclusion and collapsing of phases}

Instances in the type hierarchy can be part of a phase-level mutual exclusion cluster ( $\rho$-mex cluster). The + node of every entity in a $\rho$-mex cluster sends 
inhibitory links to, and receives inhibitory links from, the + node of all other entities in the cluster. As a result of this mutual inhibition, only the most active entity within a $\rho$-mex cluster can remain active in any given phase. A similar $\rho$-mex cluster can be formed by $+e$ : nodes of mutually exclusive types as well as $+v$ : nodes of mutually exclusive types.

Another form of inhibitory interaction between siblings in the type hierarchy leads to an "explaining away" phenomenon in SHRUTI. Let us illustrate this inhibitory interaction with reference to the type hierarchy shown in Figure 1. The link from + :John to +e:Human sends an inhibitory modifier to the link from ?e:Human to ?: Mary. Similarly, the link from +:Mary to +e:Human sends an inhibitory modifier to the link from ?e:Human to ?: John (such modifiers are not shown in the figure). Analogous connections exist between all siblings in the type hierarchy. As a result of such inhibitory modifiers, if ?e:Human propagates activity to ?:John and ?:Mary in phase $\rho 1$, then the strong activation of $+:$ John in phase $\rho 1$ attenuates the activity arriving from ?e:Human into ?:Mary. In essence, the success of the query "Is it John?" in the context of the query "Is it human?" makes the query "Is it Mary?" unimportant. This use of inhibitory connections for explaining away is motivated by [2].

As discussed in Section 8, SHRUTI supports the introduction of "new" phases during inference. In addition, SHRUTi also allows multiple phases to coalesce into a single phase during inference. In the current implementation, such phase unification can occur under two circumstances. First, phase collapsing can occur whenever a single entity dominates multiple phases (for example, if the same entity comes to be the answer of multiple queries). Second, phase collapsing can occur if two unifiable instantiations of a relation arise within a focal-cluster. For example, an assertion own(Mary, Book-17) alongside the query $\exists x: B o o k$ own(Mary, $x$ )? (Does Mary own a book") will result in a merging of the two phases for "a book" and "Book-17. Note that the type hierarchy will map the query $\exists x$ :Book own(Mary, $x$ )? into own(Mary,Book-17)?, and hence, lead to a direct match between own(Mary,Book-17) and own(Mary,Book-17)?

\section{Priming: associative short-term potentiation of weights}

Let $I$ be an instance of $T 1$. If ?:I receives activity from ?e:T1 and concurrent activity from $+: I$, then the weight of the link from ?e:T1 to ?:I increases (i.e., gets potentiated) for a short-duration. ${ }^{8}$ Let $T 2$ be a supertype of $T 1$. If $? e: T 1$ receives activity from ?e:TQ, and concurrent activity from $+e: T 1$, then the weight of the link from ?e:T2 to ?e:T1 also increases for a short-duration. Analogous weight increases can occur along the link from ?v:T1 to ?v:T2 if ?v:T2 receives concurrent activity from $+v: T 2$ and ? $v: T 1$. Similarly, the weight of the link from ?:I to ?v:T1 can undergo a short-term increase if ? $v: T 1$ receives concurrent activity from $+v: T 1$ and $?: I^{9}$

\footnotetext{
${ }^{8}$ This is modeled after the biological phenomena of short-term potentiation (STP) [6].

${ }^{9}$ In principle, short-term weight increases can occur along the link from te:T1 to $+e: T 2$ if $+e: T 2$ receives concurrent activity from $+v: T 2$ and $+e: T 1$. Similarly, the
} 
The potentiation of link weights can affect the system's response time as well as the response itself. Let us refer to an entity whose incoming links are potentiated as a "primed" entity. Since a primed entity would become active sooner than an unprimed entity, a query whose answer is a primed entity would be answered faster (all else being equal). Furthermore, all else being equal, a primed entity would dominate an unprimed entity in a $\rho$-mex cluster, and hence, if a primed and an unprimed entity compete to be the filler of a role, the primed entity would emerge as the role-filler.

\section{Facts in long-term memory: E-facts and T-facts}

Currently sHruti encodes two types of relational instances (i.e., facts) in its long-term memory (LTM): episodic facts (E-Facts) and taxon facts (T-facts). While an E-fact corresponds to a specific instance of a relation, a T-fact corresponds to a distillation or statistical summary of various instances of a relation (e.g., "Days tend to be hot in June"). An E-fact $E_{1}$ associated with a relation $P$ becomes active whenever all the dynamic bindings specified in the currently active instantiation of $P$ match those encoded in $E_{1}$. Thus an E-fact is sensitive to any mismatch between the bindings it encodes and the currently active dynamic bindings. In contrast, a T-fact is sensitive only to matches between its bindings and the currently active dynamic bindings. Note that both $\mathrm{E}-$ and $\mathrm{T}-$ facts tolerate missing bindings, and hence, respond to partial cues. The encoding of E-facts is described below - the encoding of T-facts is described in [20].

Figure 5 illustrates the encoding of E-facts love(John, Mary) and $\neg$ love(Tom, Susan). Each E-fact is encoded using a distinct fact node (these are labeled F1 and F 2 in Figure 5). A fact node sends a link to the + or - collector of the relation depending on whether the fact encodes a positive or a negative assertion.

Given the query love(John,Mary)? the E-fact node F1 will become active and activate + love, $+:$ John and + :Mary nodes indicating a "yes" answer to the question. Similarly, given the query love(Tom,Susan)?, the E-fact node F2 will become active and activate -:love, +:Tom and +:Susan nodes indicating a "no" answer to the query. Finally, given the query love(John,Susan)?, neither + love nor -:love would become active, indicating that the system can neither affirm nor deny whether John loves Susan (the nodes $+:$ John and $+:$ Susan will also not receive any activation).

Types can also serve as role-fillers in E-facts (e.g., Dog in "Dogs chase cats") and so can unspecified instances of a type (e.g., a dog in "a dog bit John"). Such E-facts are encoded by using the appropriate nodes in the focal-cluster for "Dog". In general, if an existing instance, $I$, is a role-filler in a fact, then ?:I provides the input to the fact cluster and $+: I$ receives inputs from the binder

node in the fact cluster. If the whole type $T$ is a role-filler in a fact, then ?v:T provides the input to the fact cluster and $+v: T$ receives inputs from the binder node in the fact cluster. If an unspecified instance of type $T$ is a role-filler in a

weight of the link from $+: I$ to $+e: T 1$ can undergo a short-term increase, if $+e: T 1$ receives concurrent activity from $+v: T 1$ and $+: I$. 
long-term fact, then a new instance of type $T$ is created and its "?" and " + " nodes are used to encode the fact.

(a)

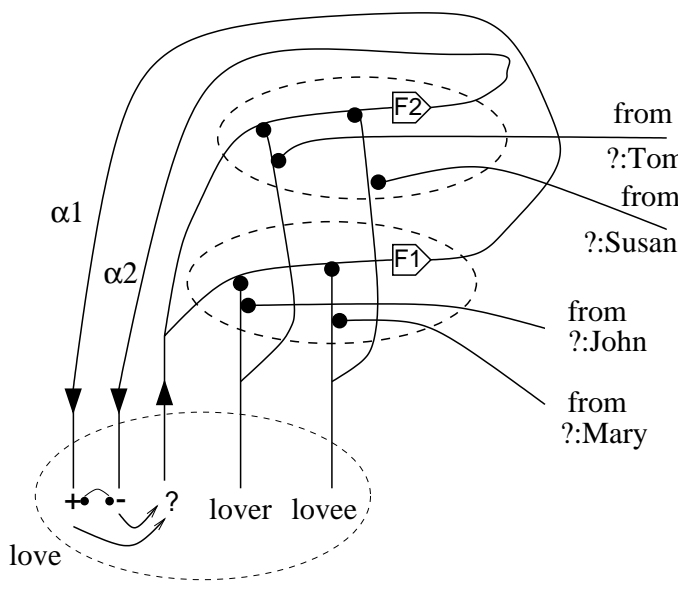

(b)

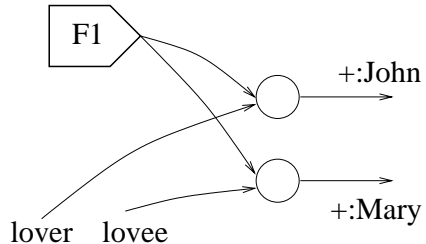

Fig. 5. (a) The encoding of E-facts: love(John,Mary) and ᄀlove(Tom,Susan). The pentagon shaped nodes are "fact" nodes and are of type $\tau$-and. The dark blobs denote inhibitory modifiers. The firing of a role node without the synchronous firing of the associated filler node blocks the activation of the fact node. Consequently, the E-fact is blocked whenever there is a mismatch between the dynamic binding of a role and its binding specified in the E-fact. (b) Links from the fact node back to role-fillers are shown only for the fact love (John, Mary) to avoid clutter. The circular nodes are $\mathrm{m}-\rho$ nodes with a high threshold which is satisfied only when both the role node and the fact node are firing. Consequently, a binder node fires in phase with the associated role node, if the fact node is firing. Weights $\alpha 1$ and $\alpha 2$ indicate strengths of belief.

\section{Encoding of rules}

A rule is encoded via a mediator focal-cluster that mediates the flow of activity and bindings between antecedent and consequent clusters (mediators are depicted as parallelograms in Figure 1). A mediator consists of a single collector $(+)$, an enabler (?), and as many role-instantiation nodes as there are distinct variables in the rule. A mediator establishes links between nodes in the antecedent and consequent clusters as follows: (i) The roles of the consequent and antecedent relation(s) are linked via appropriate role-instantiation nodes in the mediator. This linking reflects the correspondence between antecedent and consequent roles specified in the rule. (ii) The enabler of the consequent is connected to the enabler of the antecedent via the enabler of the mediator. (iii) The appropriate $(+/-)$ collector of the antecedent relation is linked to the appropriate 
$(+/-)$ collector of the consequent relation via the collector of the mediator. A collector to collector link originates at the $+(-)$ collector of an antecedent relation if the relation appears in its positive (negated) form in the antecedent. The link terminates at the $+(-)$ collector of the consequent relation if the relation appears in a positive (negated) form in the consequent. ${ }^{10}$

Consider the encoding of the following rule in Figure 1:

$\forall x$ :agent y:agent $z$ :thing give $(x, y, z) \Rightarrow$ own $(y, z)[800,800]$

This rule is encoded via the mediator, med 1 , containing three role-instantiation nodes $r 1, r 2$, and $r 3$. The weight on the link from ?:med1 to ?:give indicates the degree of evidential support for give being the probable cause (or explanation) of own. The weight on the link from + :med 1 to + :own indicates the degree of evidential support for own being a probable effect of give. These strengths are defined on a non-linear scale ranging from 0 to 1000.

A role-instantiation node is an abstraction of a neural circuit with the following functionality. If a role-instantiation node receives activation from the mediator enabler and one or more consequent role nodes, it simply propagates the activity onward to the connected antecedent role nodes. If on the other hand, the role-instantiation node receives activity only from the mediator enabler, it sends activity to the ?e node of the type specified in the rule as the type restriction for this role. This causes the $e_{e}$ node of this type to become active in an unoccupied phase. ${ }^{11}$ The $? e$ node of the type conveys activity in this phase to the role-instantiation node which in turn propagates this activity to connected antecedent roles nodes. The links between role-instantiation nodes and nodes in the type hierarchy has not been shown in Figure 1.

SHRUTI can encode rules involving multiple antecedents and consequents (see [20]). Furthermore, SHRUTi allows a bounded number of instantiations of the same predicate to be simultaneously active during inference [14].

\section{An example of inference}

Figure 2 depicts a schematized response of the SHRUTI network shown in Figure 1 to the query "Does Mary own a book?" ( $\exists x$ :Book own(Mary, $x)$ ?). This query is posed by activating?:Mary and ?e:book nodes, the role nodes owner and o$o b j$, and the enabler ?:own, as shown in Figure 2. We will refer to the phases of activation of ?:Mary and ?e:book as $\rho 1$ and $\rho 2$, respectively. Activation from the focal-cluster for own reaches the mediator structure of rules (1) and (2). Consequently, nodes $r 2$ and $r 3$ in the mediator med 1 become active in phases $\rho 1$ and $\rho 2$, respectively. Similarly, nodes $s 1$ and $s 2$ in the mediator med 2 become active in phases $\rho 1$ and $\rho 2$, respectively. At the same time, the activation from ?:own activates the enablers?:med 1 and ?:med 2 in the two mediators. Since $r 1$

${ }^{10}$ The design of the mediator was motivated, in part, by discussions the author had with Jerry Hobbs.

11 A similar phase-allocation mechanism is used in [1] for realizing function terms. Currently, an unoccupied phase is assigned in software, but eventually this will result from inhibitory interactions between nodes in the type hierarchy. 
does not receive activation from any of the roles in its consequent's focal-cluster (own), it activates the node? e:agency in the type hierarchy in a free phase (say $\rho 3)$.

The activation from nodes $r 1, r 2$ and $r 3$ reach the roles giver, recip and $g$-obj in the give focal-cluster, respectively. Similarly, activation from nodes $s 1$ and $s \mathscr{\sim}$ reach the roles buyer and $b$-obj in the buy focal-cluster, respectively. In essence, the system has created new bindings for give and buy wherein giver is bound to an undetermined agent, recipient is bound to Mary, $g$-obj is bound to a book, buyer is bound to Mary, and $b$-obj is bound to a book. These bindings together with the activation of the enabler nodes ?:give and ?:own encode two new queries: "Did some agent give Mary a book?" and "Did Mary buy a book?".

At the same time, activation travels in the type hierarchy and thereby maps the query to a large number of related queries such as "Did a human give Mary a book?", "Did John give Mary Book-17?", "Did Mary buy all books" etc.

The E-fact give(John, Mary, Book-17) now becomes active as a result of matching the query give(John, Mary, Book-17)? and causes +:give to become active. This in turn causes $+: m e d 1$, to become active and transmit activity to + :own. This results in an affirmative answer to the query and creates a reverberant loop of activity involving the clusters own, med1, give, the fact node F1, and the entities John, Mary, and Book-1\%.

\section{Conclusion}

The type structure described above, together with other enhancements such as support for negation, priming, and evidential rules, allow SHRUTi to support a rich set of inferential behaviors, and perhaps, shed some light on the nature of symbolic neural representations.

SHRUTI identifies a number of constraints on the representation and processing of relational knowledge and predicts the capacity of the active (working) memory underlying reflexive reasoning [17][22]. First, on the basis of neurophysiological data pertaining to the occurrence of synchronous activity in the $\gamma$ band, SHRUTi leads to the prediction that a large number of facts (relational instances) can be active simultaneously and a large number of rules can fire in parallel during an episode of reflexive reasoning. However, the number of distinct entities participating as role-fillers in these active facts and rules must remain very small $(\approx 7)$. Recent experimental findings as well as computational models lend support to this prediction (e.g., [12][13]). Second, since the quality of synchronization degrades as activity propagates along a chain of cell clusters, SHRUTI predicts that as the depth of inference increases, binding information is gradually lost and systematic inference reduces to a mere spreading of activation. Thus SHRUTI predicts that reflexive reasoning has a limited inferential horizon. Third, SHRUTi predicts that only a small number of instances of any given relation can be active simultaneously.

A number of issues remain open. These include the encoding of rules and facts involving complex nesting of quantifiers. While the current implementation 
supports multiple existential and universal quantifiers, it does not support the occurrence of existential quantifiers within the scope of an universal quantifier. Also, the current implementation does not support the encoding of complex types such as radial categories [10]. Another open issue is the learning of new relations and rules (mappings). In [18] it is shown that a recurrent network can learn rules involving variables and semantic restrictions using gradient-descent learning. While this work serves as a proof of concept, it does not address issues of scaling and catastrophic interference. Several researchers are pursuing solutions to the problem of learning in the context of language acquisition (e.g., [16][4][7]).

In collaboration with M. Cohen, B. Thompson, and C. Wendelken, the author is also augmenting SHRUTI to integrate the propagation of belief with the propagation of utility. The integrated system will be capable of seeking explanations, making predictions, instantiating goals, constructing reactive plans, and triggering actions that maximize the system's expected future utility.

\section{Acknowledgment}

This work was partially funded by grants NSF SBR-9720398 and ONR N0001493-1-1149, and subcontracts from Cognitive Technologies Inc. related to contracts ONR N00014-95-C-0182 and ARI DASW01-97-C-0038. Thanks to M. Cohen, J. Feldman, D. Grannes, J. Hobbs, D.R. Mani, B. Thompson, and C. Wendelken.

\section{References}

1. Ajjanagadde, V.: Reasoning with function symbols in a connectionist network. In the Proceedings of the 12th Conference of the Cognitive Science Society, Cambridge, MA. (1990) 285-292.

2. Ajjanagadde, V.: Abductive reasoning in connectionist networks: Incorporating variables, background knowledge, and structured explanada, Technical Report WSI 91-6, Wilhelm-Schickard Institute, University of Tubingen, Germany (1991).

3. Ajjanagadde, V., Shastri, L.: Efficient inference with multi-place predicates and variables in a connectionist network. In the Proceedings of the 11th Conference of the Cognitive Science Society, Ann-Arbor, MI (1989) 396-403.

4. Bailey, D., Chang, N., Feldman, J., Narayanan, S.: Extending Embodied Lexical Development. In the Proceedings of the 20th Conference of the Cognitive Science Society, Madison, WI. (1998) 84-89.

5. Barnden, J., Srinivas, K.: Encoding Techniques for Complex Information Structures in Connectionist Systems. Connection Science, 3, 3 (1991) 269-315.

6. Bliss, T.V.P., Collingridge, G.L.: A synaptic model of memory: long-term potentiation in the hippocampus. Nature 361, (1993) 31-39.

7. Gasser, M., Colunga, E.: Where Do Relations Come From? Indiana University Cognitive Science Program, Technical Report 221, (1998).

8. Hobbs, J.R., Stickel, M., Appelt, D., Martin, P.: Interpretation as Abduction, Artificial Intelligence, 63, 1-2, (1993) 69-142.

9. Hummel, J. E., Holyoak, K.J.: Distributed representations of structure: a theory of analogical access and mapping. Psychological Review, 104, (1997) 427-466. 
10. Lakoff, G.: Women, Fire, and Dangerous Things - What categories reveal about the mind, University of Chicago Press, Chicago (1987).

11. Lange, T. E., Dyer, M. G.: High-level Inferencing in a Connectionist Network. Connection Science, 1, 2 (1989) 181-217.

12. Lisman, J. E., Idiart, M. A. P.: Storage of $7 \pm 2$ Short-Term Memories in Oscillatory Subcycles. Science, 267 (1995) 1512-1515.

13. Luck, S. J., Vogel, E. K.: The capacity of visual working memory for features and conjunctions. Nature 390 (1997) 279-281.

14. Mani, D.R., Shastri, L.: Reflexive Reasoning with Multiple-Instantiation in a Connectionist Reasoning System with a Typed Hierarchy, Connection Science, 5, 3\&4, (1993) 205-242.

15. Park, N.S., Robertson, D., Stenning, K.: An extension of the temporal synchrony approach to dynamic variable binding in a connectionist inference system. Knowledge-Based Systems, 8, 6 (1995) 345-358.

16. Regier, T.: The Human Semantic Potential: Spatial Language and Constrained Connectionism, MIT Press, Cambridge, MA, (1996).

17. Shastri, L.: Neurally motivated constraints on the working memory capacity of a production system for parallel processing. In the Proceedings the 14 th Conference of the Cognitive Science Society, Bloomington, IN (1992) 159-164.

18. Shastri, L.: Exploiting temporal binding to learn relational rules within a connectionist network. TR-97-003, International Computer Science Institute, Berkeley, CA, (1997).

19. Shastri, L.: A Model of Rapid Memory Formation in the Hippocampal System, In the Proceedings of the 19th Annual Conference of the Cognitive Science Society, Stanford University, CA, (1997) 680-685.

20. Shastri, L.: Advances in SHRUTI - A neurally motivated model of relational knowledge representation and rapid inference using temporal synchrony. Applied Intelligence, 11 (1999) 79-108.

21. Shastri, L.: Recruitment of binding and binding-error detector circuits via longterm potentiation. Neurocomputing, 26-27 (1999) 865-874.

22. Shastri, L., Ajjanagadde V.: From simple associations to systematic reasoning: A connectionist encoding of rules, variables and dynamic bindings using temporal synchrony. Behavioral and Brain Sciences, 16:3 (1993) 417-494.

23. Shastri, L., Grannes, D.J.: A connectionist treatment of negation and inconsistency. In the Proceedings of the 18th Conference of the Cognitive Science Society. San Diego, CA, (1996).

24. Shastri, L., Grannes, D.J., Narayanan, S., Feldman, J.A.: A Connectionist Encoding of Schemas and Reactive Plans. In Hybrid Information Processing in Adaptive Autonomous vehicles, G.K. Kraetzschmar and G. Palm (Eds.), Lecture Notes in Computer Science, Springer-Verlag, Berlin (To appear).

25. Shastri, L., Wendelken, C.: Knowledge Fusion in the Large - taking a cue from the brain. In the Proceedings of the Second International Conference on Information Fusion, FUSION'99, Sunnyvale, CA, July (1999) 1262-1269.

26. Singer, W.: Synchronization of cortical activity and its putative role in information processing and learning. Annual Review of Physiology 55 (1993) 349-74.

27. Sun, R.: On variable binding in connectionist networks. Connection Science, 4, 2 (1992) 93-124.

28. von der Malsburg, C.: Am I thinking assemblies? In Brain Theory, ed. G. Palm \& A. Aertsen. Springer-Verlag (1986). 